\title{
Toward in vivo dosimetry in external beam radiotherapy using x-ray acoustic computed tomography: A soft-tissue phantom study validation
}

\author{
Hao Lei \\ Department of Mechanical Engineering, University of Michigan, 2300 Hayward Street, Ann Arbor, MI 48109, USA
}

Wei Zhang

Department of Biomedical Engineering, University of Michigan, 2200 Bonisteel Boulevard, Ann Arbor, MI 48109, USA

Institute of Biomedical Engineering, Chinese Academy of Medical Sciences \& Peking Union Medical College, 236 Baidi Rd, Nankai District, Tianjin, China

Ibrahim Oraiqat

Department of Radiation Oncology, University of Michigan, 519 W. William St, Argus Bldg. 1, Ann Arbor, 48103-4943 MI, USA

Zhipeng Liu

Institute of Biomedical Engineering, Chinese Academy of Medical Sciences \& Peking Union Medical College, 236 Baidi Rd, Nankai District, Tianjin, China

Jun $\mathrm{Ni}$

Department of Mechanical Engineering, University of Michigan, 2300 Hayward Street, Ann Arbor, MI 48109, USA

Xueding Wang ${ }^{\text {a) }}$

Department of Biomedical Engineering, University of Michigan, 2200 Bonisteel Boulevard, Ann Arbor, MI 48109, USA

Issam El Naqaa)

Department of Radiation Oncology, University of Michigan, 519 W. William St, Argus Bldg. 1, Ann Arbor, 48103-4943 MI, USA

(Received 12 February 2018; revised 14 May 2018; accepted for publication 22 June 2018; published 24 July 2018)

Purpose: To study, using phantoms made from biological tissues, the feasibility and practical challenges of monitoring the position of the radiation beam and the deposited dose by x-ray acoustic computed tomography (XACT) during external beam radiotherapy delivery.

Material and methods: A prototype XACT system with a single immersion ultrasound transducer, which was positioned around the target sample driven by a motor-controlled rotation stage, was used to acquire the X-ray acoustic (XA) signals produced by a medical linear accelerator (Linac) to form an XACT image of the irradiated phantom. To investigate the feasibility of XACT in tracking the position of radiation dose, a large piece of veal liver with embedded fat tissue was imaged and beam misalignments were measured. Next, we explored the sensitivity of XACT in monitoring and quantifying the delivered dose, in which a block of porcine gel was embedded with equally spaced lard cylinders and imaged. The doses on the lard cylinders modulated by physical wedges were quantified from the XACT image and were verified by comparison to measurements from radiochromic films as the gold standard. Then, to simulate how XACT can perform in a targeted tissue in the human body, a porcine gel phantom with lard cylinders covered by different materials (bone, muscle, and air gap, respectively) was also imaged.

Results: The reconstructed XACT images of the phantoms show congruence with the boundaries of the beam field and the interfaces between the different tissue materials. The beam displacement from the target was tracked properly by the reconstructed XACT images. An intensity difference as small as $2.9 \%$ in delivered dose region can be measured from XACT images $P=0.02$. The intensities of XACT images were highly correlated to the film measurements with an $R^{2}$ better than 0.986 . The expected variances of dose delivered to different target regions as a result of the difference in attenuation were successfully captured by the XACT images.

Conclusions: This study validated the feasibility of XACT in accurately obtaining relative dose maps of tissue-mimicking phantoms. XACT offers a practical method for verifying the beam position against the target and quantifying the relative dose delivered to the target during external beam radiotherapy. () 2018 American Association of Physicists in Medicine [https://doi.org/10.1002/mp.13070]

Key words: photoacoustic imaging, radiation dosimetry, radiation therapy, ultrasound imaging, x-ray acoustic computed tomography 


\section{INTRODUCTION}

As one of the main treatment options for cancer, radiation therapy is received by more than half of all cancer patients. ${ }^{1}$ It works by damaging the DNA of the cancer cells using high-energy beams of ionizing radiation., ${ }^{2,3}$ Primarily, clinical linear accelerators (Linac), which produce high-energy megavoltage photon or electron beams, are used to deliver ionizing radiation to patients from outside of the body during external beam radiotherapy (EBRT). Radiation damages affect not only cancerous tissues but also healthy cells. Therefore, the premise of radiation therapy is to optimize a strategy for maximizing the damage to cancer cells while minimizing the damage to the surrounding healthy regions. The demand for delivering the correct dose to the proper location inside patient's body leads to more advanced treatment delivery techniques such as intensity modulated radiation therapy (IMRT) and volumetric-modulated arc therapy (VMAT). ${ }^{4-7}$ However, the complication due to organ deformations and respiratory motion still cannot be avoided. ${ }^{8,9}$ Therefore, involving a volumetric form of in vivo dose measurement is necessary to assess clinically relevant differences in the delivered intensity and delivery location between the planned and the delivered radiation dose.

Several techniques for real-time in vivo dosimetry are currently used to monitor the radiation dose delivery during EBRT, ${ }^{10,11}$ including silicon diodes, ${ }^{12,13}$ metal oxide semiconductor field effect transistors (MOSFETs), ${ }^{14,15}$ and electronic portal imaging devices (EPIDs). ${ }^{16,17}$ However, the silicon diodes and MOSFETs are point dosimeters, and the EPIDs are not accurate enough due to their exit dose nature and lack of sufficient sensitivity. ${ }^{11,18}$

Since the pulsed radiation beam produced by Linacs generates transient temperature rise in tissue after being absorbed, similar to photoacoustic imaging (PAI), ${ }^{19}$ the acoustic waves induced by the high-energy $\mathrm{x}$ rays, namely $\mathrm{x}$-ray acoustic (XA) wave, could be detected by ultrasonic transducers. Recently, the detection of XA signals in clinical Linacs has been demonstrated, and the XA computed tomography (XACT) imaging of a lead rod embedded in chicken breast tissue has been achieved. ${ }^{20}$ Lately, the ability to form experimental XACT images in pure water in a variety of clinically relevant situations and to extract accurate dosimetric information from such images was also demonstrated. ${ }^{21,22}$ Besides the studies working with highdose $\mathrm{x}$ ray generated by Linacs, XACT imaging based on the $\mathrm{kV}$ X-ray excitations has been explored. ${ }^{23,24}$ These results inspire us to further develop and assess the performance of XACT using more realistic soft-tissue phantoms. The goal of this study is to explore the feasibility of $\mathrm{XACT}$ in providing relevant feedback information by evaluating (a) the relative radiation dose delivered to the target tissue and (b) the dose delivery position in relation to the target tissue.

\section{MATERIALS AND METHODS}

\section{A. X-ray-induced acoustic wave generation}

In the process of $\mathrm{x}$-ray absorption, the MVs x-ray photon produced by Linacs interacts with an outer-shell orbital electron of an atom primarily through the Compton effect. ${ }^{25}$ The photon is scattered at some angle with reduced energy, while the orbital electron is ejected with kinetic energy equal to the difference between the energy of the incident and scattered photon. The scattered electron, now called the Compton electron, will propagate and scatter inside the medium and finally be reabsorbed to produce heat with a thermal efficiency $\eta_{t h}{ }^{26}$ :

$$
H(\boldsymbol{r}, t)=\eta_{t h} \rho D_{r}(\boldsymbol{r}, t)
$$

where $H(\boldsymbol{r}, t)$ is the heat absorption rate at location $\boldsymbol{r} \in \boldsymbol{R}^{\mathbf{3}}$ and time $t, \rho$ is the mass density of the material, and $D_{r}(\boldsymbol{r}, t)$ is the temporal dose rate during a single $\mathrm{x}$-ray pulse. The temperature rise distribution $T(\boldsymbol{r}, t)$ due to the heat source term $H(\boldsymbol{r}, t)$ follows the equation ${ }^{27}$ :

$$
\rho C_{v} \frac{\partial T(\boldsymbol{r}, t)}{\partial t}=\lambda \nabla^{2} T(\boldsymbol{r}, t)+H(\boldsymbol{r}, t)
$$

where $C_{v}$ is specific heat capacity and $\lambda$ is the thermal conductivity. During a single x-ray pulse, the thermal diffusion distance $d_{t h}$ can be evaluated by:

$$
d_{t h}=2 \sqrt{\alpha_{t h} \tau_{p}}
$$

where $\alpha_{t h}$ is the thermal diffusivity $\left(\sim 0.15 \mathrm{~mm}^{2} / \mathrm{s}\right.$ for soft tissue), ${ }^{28}$ and $\tau_{p}$ is the pulse duration which is around 3-6 $\mu$ s for clinic Linacs. ${ }^{29,30}$ The thermal diffusion distance is less than $2 \mu \mathrm{m}$ during the excitation pulse, which is much smaller than the scale of spatial resolution considered in this study, and hence negligible. Therefore, Eq. (2) regarding temperature rise can be rewritten with the temporal dose rate by omitting the first term on the right-hand side:

$$
\frac{\partial T(\boldsymbol{r}, t)}{\partial t}=\frac{\eta_{t h} D_{r}(\boldsymbol{r}, t)}{C_{v}}
$$

Hence, the wave equation governing the generation and propagation of the x-ray-induced acoustic pressure due to the temperature rise given by ${ }^{31}$ :

$$
\left(\nabla^{2}-\frac{1}{v_{s}^{2}} \frac{\partial^{2}}{\partial t^{2}}\right) p(\boldsymbol{r}, t)=-\frac{\beta B}{v_{s}^{2}} \frac{\partial^{2} T(\boldsymbol{r}, t)}{\partial t^{2}}
$$

can be rewritten as follows:

$$
\left(\nabla^{2}-\frac{1}{v_{s}^{2}} \frac{\partial^{2}}{\partial t^{2}}\right) p(\boldsymbol{r}, t)=-\frac{\beta K_{T} \eta_{t h}}{v_{s}^{2} C_{v}} \frac{\partial D_{r}(\boldsymbol{r}, t)}{\partial t}
$$

where $v_{s}=\sqrt{K_{T} / \rho}$ is the velocity of sound, $\beta$ is the volumetric thermal expansion coefficient, and $K_{T}$ is the isothermal bulk modulus. The solved XA pressure detected at the transducer position $\boldsymbol{r}$ and time $t$ can be expressed by:

$$
p(\boldsymbol{r}, t)=\left.\frac{1}{4 \pi v_{s}^{2}} \int d \boldsymbol{r}^{\prime} \frac{1}{\left|\boldsymbol{r}-\boldsymbol{r}^{\prime}\right|} \Gamma \eta_{t h} \rho \frac{\partial D_{r}\left(\boldsymbol{r}^{\prime}, t^{\prime}\right)}{\partial t^{\prime}}\right|_{t^{\prime}=t-\frac{\left|r-r_{s}^{\prime}\right|}{v_{s}}}
$$


where $\Gamma$ is the Grüneisen parameter defined as follows:

$$
\Gamma=\frac{\beta K_{T}}{C_{v} \rho}
$$

\section{B. Dose information from $x$-ray acoustic images}

Unlike the pulsed laser applied for PA imaging, clinic Linacs offer the excitation source with relatively long pulse duration of 3-6 $\mu$ s, which makes it hard to satisfy the stress confinement $\left(\tau_{p} \cdot v_{s} \ll\right.$ spatial resolution). ${ }^{19,31,32}$ By selecting a low-frequency acoustic detection range, which leads to lower spatial resolution, the temporal profile of a single $\mathrm{x}$-ray pulse can be treated as a Dirac delta function and Eq. (7) can be rewritten as follows:

$$
p(\boldsymbol{r}, t)=\frac{1}{4 \pi v_{s}^{2}} \frac{\partial}{\partial t} \int d \boldsymbol{r}^{\prime} \frac{1}{\left|\boldsymbol{r}-\boldsymbol{r}^{\prime}\right|} \frac{\Gamma \eta_{t h} \rho}{\tau_{p}} D_{p}\left(\boldsymbol{r}^{\prime}\right) \delta\left(t-\frac{\left|\boldsymbol{r}-\boldsymbol{r}^{\prime}\right|}{v_{s}}\right)
$$

where $D_{p}(\boldsymbol{r})=D_{r}(\boldsymbol{r}, t) \tau_{p}$ is the local dose due to a single $\mathrm{x}$ ray pulse. Meanwhile, the initial acoustic pressure $p_{0}(\boldsymbol{r})$ induced by $\mathrm{x}$-ray excitation can be obtained by:

$$
p_{0}(\boldsymbol{r})=\frac{\Gamma \eta_{t h} \rho}{\tau_{p}} D_{p}(\boldsymbol{r})
$$

Even though it is challenging to resolve quantitatively accurate initial acoustic pressure $p_{0}(\boldsymbol{r})$ from the acquired XA signals $p(\boldsymbol{r}, t),{ }^{33}$ the pixel value inside the XA image is proportional to the local dose deposited per pulse. The correlation between the relative XA intensity $I(r)$ and the local deposited dose $D_{p}(\boldsymbol{r})$ can be described as follows:

$$
I(\boldsymbol{r}) \propto \frac{\Gamma \eta_{t h} \rho}{\tau_{p}} D_{p}(\boldsymbol{r})
$$

Therefore, the image of relative XA intensity $I(\boldsymbol{r})$ will reveal (a) information about beam position and field shape during the dose delivery and (b) dose amount deposited to the target volume which may undergo different attenuations.

\section{C. XACT experimental setup}

Figure 1 shows the schematic diagram and the photograph of the prototype XACT system at our institution. A clinical radiotherapy Linac (TrueBeam ${ }^{\mathrm{TM}}$, Varian Medical Systems, Palo Alto, CA, USA) provided $6 \mathrm{MV}$ photon beam pulses with $4 \mu \mathrm{s}$ pulse duration, and operated at a dose rate of $800 \mathrm{MU} / \mathrm{min}$. An unfocused immersion transducer (V301, Olympus-NDT) with central frequency of $0.5 \mathrm{MHz}$ and $-6 \mathrm{~dB}$ bandwidth of $64 \%$ was driven by a computer-controlled stepper motor (MD2, Arrick Robotics, Hurst, TX, USA) to scan around the phantom inside the water tank. The transducer was used to detect the XA waves generated from the phantom and transmitted through water at each scanning position. The output of the transducer was amplified by the preamplifier (5660B, Olympus-NDT), which was set to a gain of $60 \mathrm{~dB}$ with a bandwidth from $50 \mathrm{kHz}$ to $2 \mathrm{MHz}$, and then recorded by a 14-bit digitizer card (Razor 14, GaGe) inside a PC sited outside the treatment room. The acquisitions were triggered by the Linac target pulses sent out from the Linac console and were operating at $10-\mathrm{MHz}$ sampling frequency. To scan a 2D image, the transducer rotated along an entire circle (diameter $12 \mathrm{~cm}$ ) around the phantom with a total of 120 equiangular steps. The measurement at each scanning position was averaged over 660 pulses and the signal acquisition for a 2D image took about $10 \mathrm{~min}$. To avoid the attenuation of the radiation beam passing through the rotation stage, the gantry was rotated by $180^{\circ}$ so that the beam came from the bottom of the water tank.

\section{D. Image reconstruction}

The signals recorded at each scanning position were processed with a median filter before taking the average. A digital high-pass filter with cutoff frequency at $50 \mathrm{kHz}$ was applied to remove DC and low-frequency background drift inside the averaged signals, and a Savitzky-Golay denoising filter (order of 3, and window of 31) was applied to further smooth the signals before reconstruction. Then, 2D XACT images on the scanning plane were reconstructed based on a simple filtered back-projection algorithm. ${ }^{34}$

\section{E. Imaging of deposited dose}

To validate the feasibility of XACT in verifying the shape and the location of the x-ray field during treatment, a (a)

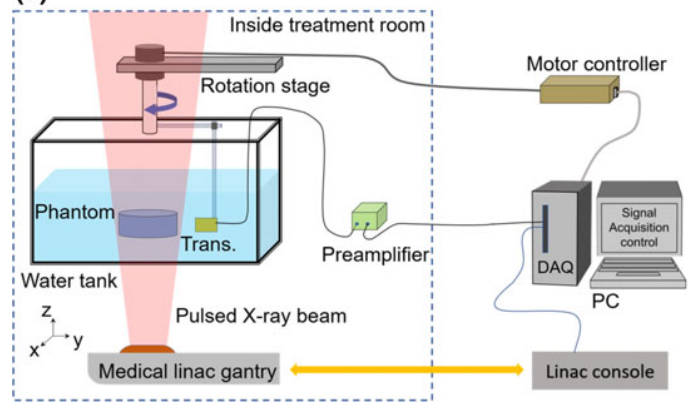

(b)

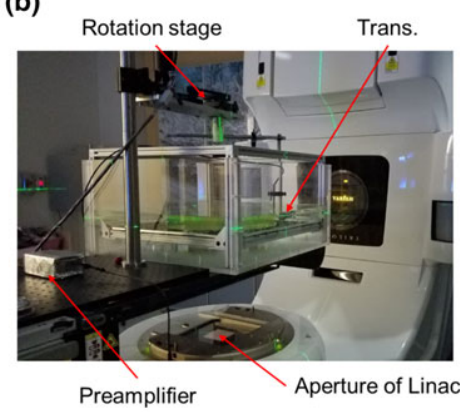

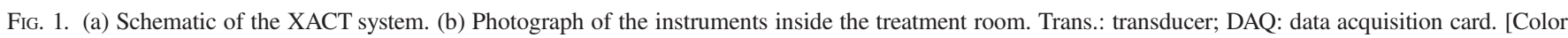
figure can be viewed at wileyonlinelibrary.com] 
phantom containing two pieces of veal liver and one cuboid of fat was prepared, as shown in Fig. 2. A cuboid of tissue was removed from the larger piece of veal liver and replaced with the cuboid of fat $(6 \times 4 \times 3 \mathrm{~cm})$ as the target of treatment. All the tissues were fixed in a block of porcine gel (10 g/100 ml, G2500, Sigma-Aldrich).

Before XACT imaging, an ultrasound (US) image of the phantom was acquired by compounding 12 US B-mode images acquired around the phantom. The $12 \mathrm{~B}$-mode images were scanned by a commercial system (Z.one, Zonare Medical Systems) and a phased array probe (P4-1, Zonare Medical Systems, Mountain view, CA, USA). To acquire images at different locations around the phantom, the probe was driven by the same rotation stage in the XACT system. With a $30^{\circ}$ step size, 12 images were scanned along an entire circle around the phantom. In the experiment, the x-ray beam had a field size of $6 \times 4 \mathrm{~cm}$, the same as the size of the fat cuboid in the imaging plane. Both the center layer of the phantom and the scanning plane of the transducer were adjusted to satisfy a source-to-surface distance (SSD) of $100 \mathrm{~cm}$. For the first XACT imaging, the x-ray beam was aligned with the position of the fat cuboid, as shown by the Field 1 in Fig. 2(b). To mimic the situation that the x-ray beam was offtarget due to body motions or misplacement errors, the $\mathrm{x}$-ray beam was shifted away from the target during the second XACT imaging, as shown by the Field 2 in Fig. 2(b).

\section{F. Dose estimation}

To evaluate the sensitivity and accuracy of the XACT system in measuring the dose delivered to the target, a phantom with a row of five cylinders (diameter: $1 \mathrm{~cm}$; height: $2 \mathrm{~cm}$ ) made with lard and fixed inside porcine gel was prepared, as shown in Fig. 3. Three physical wedges with different attenuation slopes $\left(15^{\circ}, 30^{\circ}\right.$, and $\left.45^{\circ}\right)$ were, respectively, added to the Linac aperture, and the differences in dose delivery to the five lard cylinders were measured by our XACT system. The center plane of the lard cylinders and the scanning plane of the transducer were adjusted to the same height with a

(a)

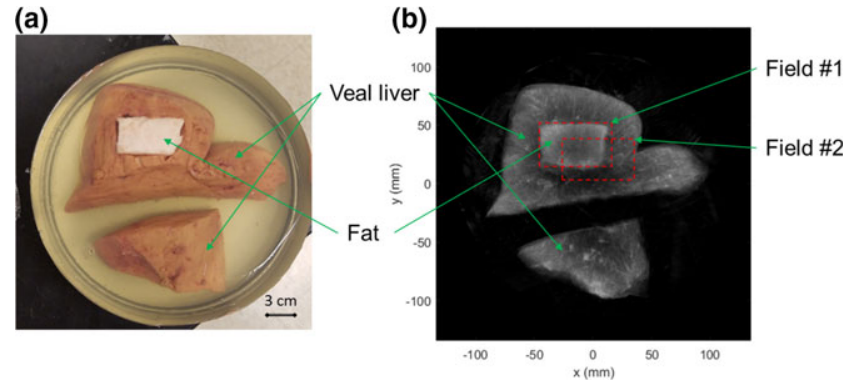

FIG. 2. (a) Photograph of a porcine gel phantom containing veal livers and fat. (b) Compounding US image of the phantom, where the shape and the location of the radiation $\mathrm{x}$-ray beam are labeled by a red dash rectangle $(6 \times 4 \mathrm{~cm})$. Field 1: the beam was aligned to the fat cuboid (i.e., target of treatment); Field 2: the beam was shifted away from the target $(20 \mathrm{~mm}$ along the $x$-axis and $-10 \mathrm{~mm}$ along the $y$-axis). [Color figure can be viewed at wile yonlinelibrary.com]
$100 \mathrm{~cm} \mathrm{SSD} \mathrm{and} 10 \mathrm{~cm}$ above the bottom of the tank. Since the five lard cylinders were prepared with the same material, the difference in absorbed dose due to the material properties can be ignored. Therefore, the intensities in the XACT image can directly reflect the local doses at the five lard cylinders. For statistical analysis, 10 independent measurements were conducted for each of the three wedges. The dose distributions at the imaging plane were also recorded by radiochromic film for comparison and validation.

Considering the complex situation inside a patient's body during treatment, the radiation dose reaching the target volume may already experience different attenuations, especially when the beam passes through covering tissues containing cavities (less attenuation) and bones (more attenuation). This is another cause of the uncertainty in estimating the dose delivered to the target. To simulate this situation and validate the feasibility of XACT in measuring the local dose delivered in such a scenario, a phantom shown in Fig. 4 was prepared and imaged. Four lard cylinders (diameter: $1 \mathrm{~cm}$; height: $2 \mathrm{~cm}$ ) were individually covered by a piece of bone (thickness: $0.9 \mathrm{~cm}$ ), an air gap (thickness: $1.5 \mathrm{~cm}$ ), a piece of pork muscle (thickness: $1.9 \mathrm{~cm}$ ), and nothing, and then all fixed inside porcine gel. Five independent measurements on this phantom were conducted for statistical analysis.

\section{RESULTS}

\section{A. Imaging of deposited dose}

The reconstructed XACT images (normalized so that the maximum intensity equals 1 ) correspondent to Field 1 (radiation beam on-target) and Field 2 (radiation beam off-target) are shown in Figs. 5(a) and 5(b), respectively. In Fig. 5(a), only the entire outline of the fat cuboid can be observed, as the whole fat cuboid was just covered by the x-ray field and the outlines of both were overlapping. In Fig. 5(b), the boundaries of the squared x-ray field can be observed, as the outline of the field was shifted off from the outline of the fat cuboid. Moreover, one corner of the fat cuboid can also be seen due to the contrast between the fat and the liver tissues. This contrast is mainly caused by the differences in Grüneisen parameter, which are 0.6 for fat and 0.15 for liver. Figures 5(c) and 5(d) show the XA images (pseudo color) superimposed on the compounding US image (gray scale). These combined images present the positions of the deposited dose (as mapped by XACT images) related to the location and shape of the treatment target (as shown by the US images).

Figures 6(a) and 6(b) display the XACT and US intensity profiles along the dash-dot lines marked in Figs. 5(c) and 5(d). The vertical line is through $x=-14 \mathrm{~mm}$. The horizontal line is through $y=35 \mathrm{~mm}$. The US intensity profiles present the boundaries of the target. According to the US profiles, the horizontal size and the vertical size of the target along the marked lines become $59 \mathrm{~mm}$ and $41 \mathrm{~mm}$ due to the distortion, respectively. The two sets of XACT intensity profiles are correspondent to Field 1 (radiation beam on-target) and Field 2 (radiation beam off-target), respectively. 
(a)

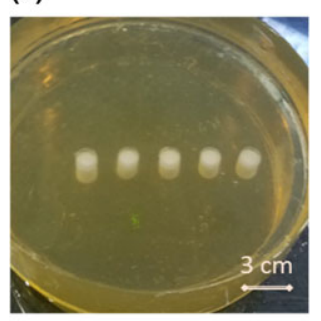

(b)

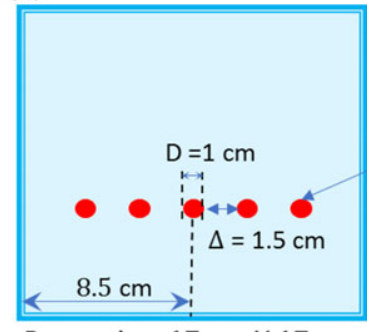

(c)

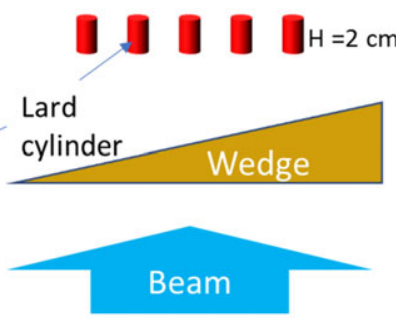

Fig. 3. (a) Photograph of a porcine gel phantom containing five identical lard cylinders distributed along a row. (b) Schematic diagram of the lard cylinders and radiation beam (top view). (c) Schematic diagram of lard cylinders, wedge, and radiation beam (side view). $D$ is the diameter of the lard cylinder; $\Delta$ is the space between two adjacent cylinders; $H$ is the height of the lard cylinder. [Color figure can be viewed at wileyonlinelibrary.com]

(a)

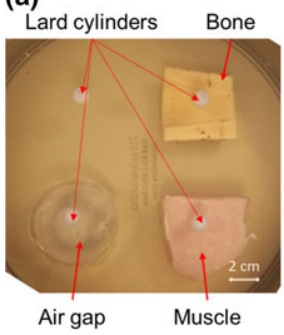

(b)

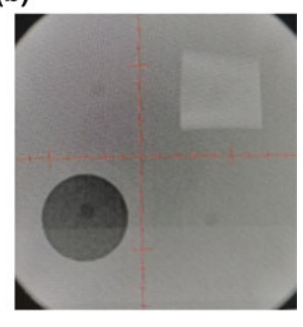

FIG. 4. (a) Photograph of a porcine gel phantom containing four identical lard cylinders covered, respectively, by different materials with different $\mathrm{x}$ ray attenuation, including bone, air gap, pork muscle, and nothing (gel only). (b) Radiograph of the phantom. [Color figure can be viewed at wileyonline library.com]
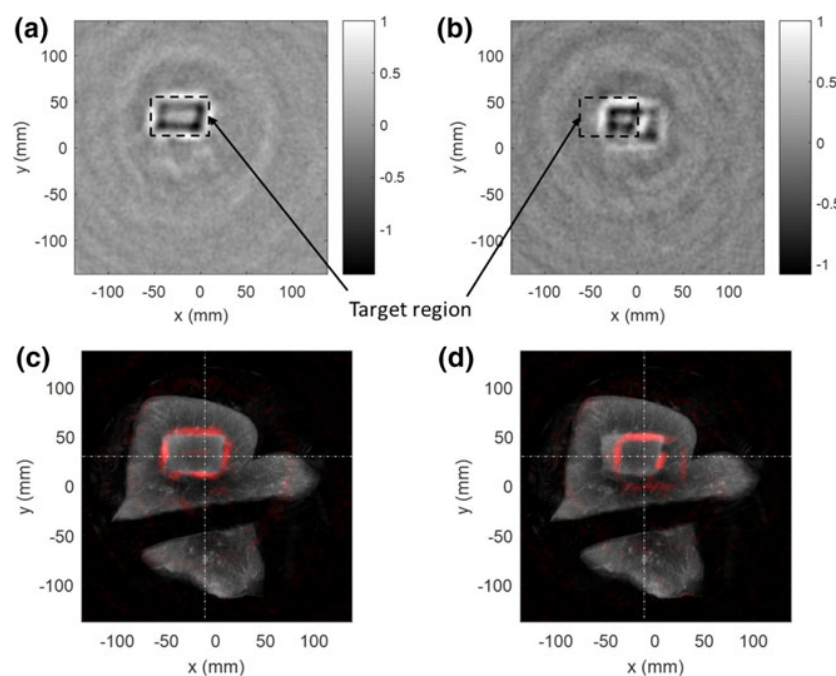

FIG. 5. Normalized XACT images corresponding to (a) Field 1 (radiation beam on-target) and (b) Field 2 (radiation beam off-target) as described in Fig. 2 (b). The dashed rectangle marks the position and the shape of the target. (c) XACT image in (a) presented in pseudo color and superimposed on the gray scale US image of the phantom. (d) XACT image in (b) presented in pseudo color and superimposed on the gray scale US image of the phantom. [Color figure can be viewed at wileyonlinelibrary.com]

Compared with the US profiles, the determined boundaries in XACT profiles labeled with green and red dash lines display the conditions of beam alignment before (on-target) and after (off-target) shifting the beam location, respectively.
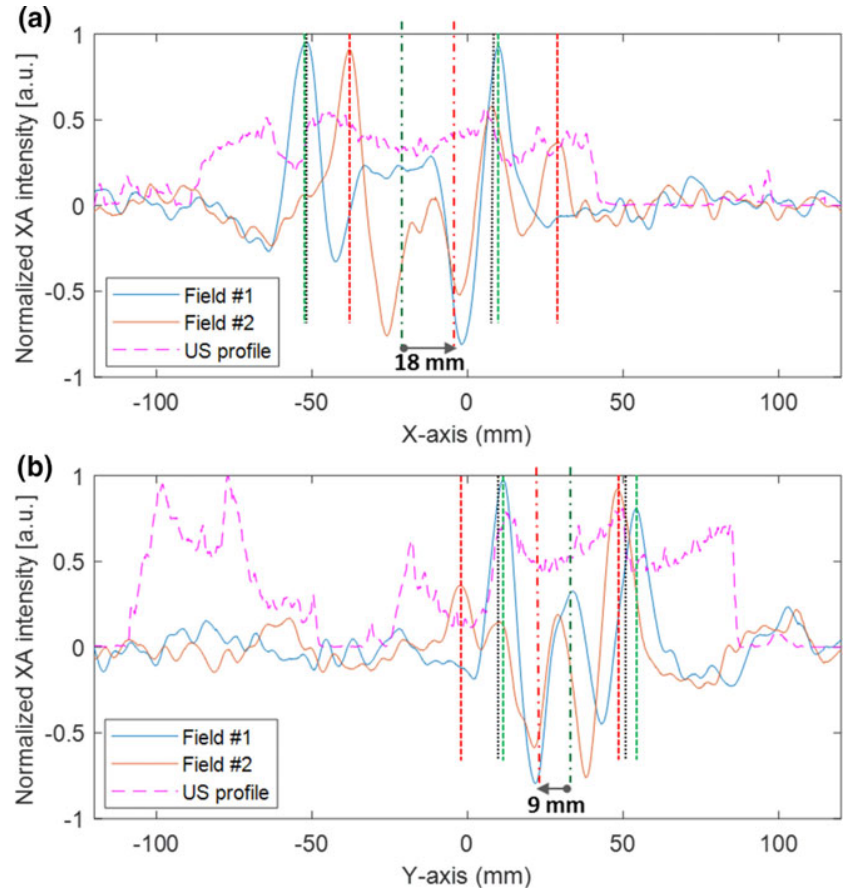

FIG. 6. The intensity profiles along (a) the marked horizontal line $(y=+35 \mathrm{~mm})$ and (b) the vertical line $(x=-14 \mathrm{~mm})$ in the XACT images of the two measurements (beam on-target and beam off-target, respectively) and the compounding US image. The green and the red dash lines label the beam boundaries determined by the profiles of XACT images before (ontarget) and after (off-target) shifting the beam location, respectively. The green and the red dash-dot lines show the centers of the beam (i.e., the central lines between the determined boundaries) before and after shifting the beam location, respectively. The gray dash lines show the boundaries of the target extracted from the intensity profiles of the US image. [Color figure can be viewed at wileyonlinelibrary.com]

Subjected to the change of the beam location, the horizontal position and the vertical position of the beam, as presented by the XACT intensity profiles, were shifted by $+18 \mathrm{~mm}$ and $-9 \mathrm{~mm}$, respectively. As shown in Figs. 5 and 6, the XA intensities peak at the interfaces, that is, the boundary of the $\mathrm{x}$-ray field or the interface between the fat cuboid and the liver. According to Eq. (9) and considering the differences between Grüneisen parameters of fat and liver, the peak of XA signal corresponding to the field boundary locating on fat is higher than the peak from the field boundary locating on liver and the peak at the fat and liver interface. 


\section{B. Dose uncertainty estimation}

Figure 7 shows XACT imaging results from the phantom containing five lard cylinders under 6-MV photon beams. The only difference between Figs. 7(a) and 7(b) is that a $30^{\circ}$ physical wedge was added to the Linac aperture for the image in (a) while no wedge was used for the image in (b). The image intensity was normalized to the maximum intensity of the image which was acquired without using the wedge. To explore the feasibility of XACT in quantifying the deposited dose in each cylinder target, the intensity profiles extracted from the two XACT images were compared in Fig. 7(c). Considering that the five cylinder targets were not perfectly aligned along a horizontal line, each intensity profile actually shows the horizontal projection of the maximum value inside the range from $y=-40 \mathrm{~mm}$ to $y=-20 \mathrm{~mm}$.

Figures $8(a)-8(c)$ present the quantified datasets of relative XA intensities for the 5 lard cylinders imaged with the use of a $15^{\circ}$ physical wedge, a $30^{\circ}$ physical wedge, and a $45^{\circ}$ physical wedge, respectively. Under each condition (i.e., three different wedges for the results in a-c, respectively), the normalized relative intensity of each of the 5 lard cylinders was quantified by dividing its maximum intensity in the XACT image by the maximum intensity of the corresponding lard cylinder from the XACT image that does not use a wedge.
Under each condition, 10 independent measurements were conducted for statistical analyses.

Figure 9(a) shows the dose profiles per pulse extracted from the film test results in the same direction as the five lard cylinders were aligned. The dose per pulse was calculated by the recorded accumulated dose dividing the monitored pulse number during each film test. For each wedge, the normalized relative XA intensities of the five lard cylinders shown in Fig. 8 are compared with the dose per pulse values read from film profiles at positions of $-50,-25,0,25$, and $50 \mathrm{~mm}$ displayed in Fig. 9(a). As derived in Section 2.B, the XA intensities $I(\boldsymbol{r})$ for each lard cylinder can be fitted to the local dose $D_{p}(\boldsymbol{r})$ measured by film with a determined constant $\xi$, which should correspond to the proportionality considered in Eq. (11):

$$
D_{p}(\boldsymbol{r})=\xi \cdot I(\boldsymbol{r})
$$

The XA intensities and the dose per pulse are plotted together in Figs. 9(b)-9(d), while Fig. 9(e) presents the proportionality between the normalized relative XA intensities and the dose per pulse by determining a constant $\xi$ for each wedge. The sensitivity of XACT imaging in monitoring the relative dose can be achieved up to $3 \%$, as the minimum dose difference between two lard cylinders under the $15^{\circ}$ physical wedge is $2.9 \%$ according to the film result shown in (a)

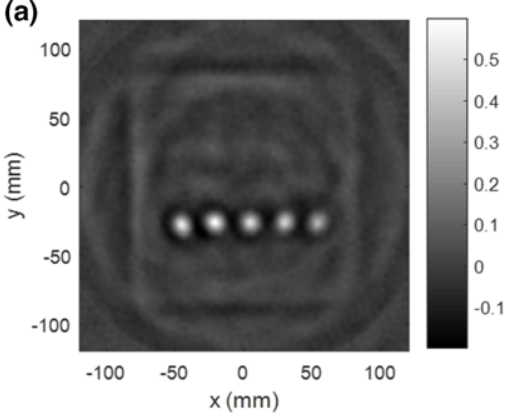

(b)

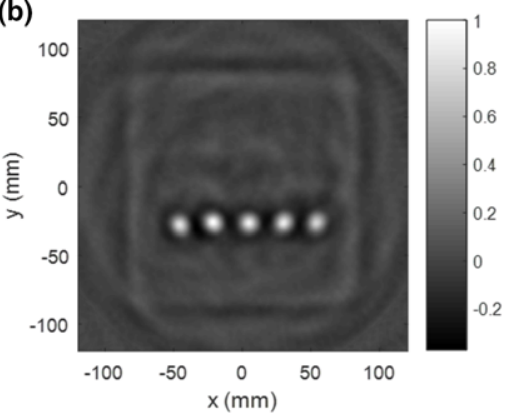

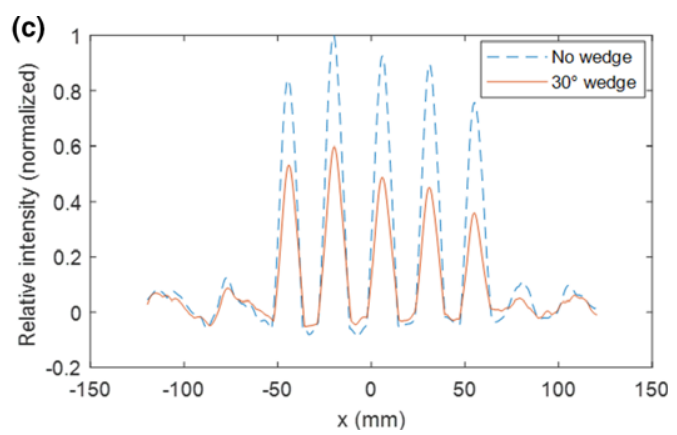

Fig. 7. (a) A reconstructed XACT image of the phantom containing five lard cylinders. A $30^{\circ}$ physical wedge was used to change the dose applied to each cylinder target. (b) A reconstructed XACT image of the same phantom where no wedge was applied. (c) The intensity profiles along the $x$-axis showing the deposited doses in the five cylinder targets for the two situations, that is, with and without using the $30^{\circ}$ wedge. [Color figure can be viewed at wileyonlinelibrary.com]

(a)

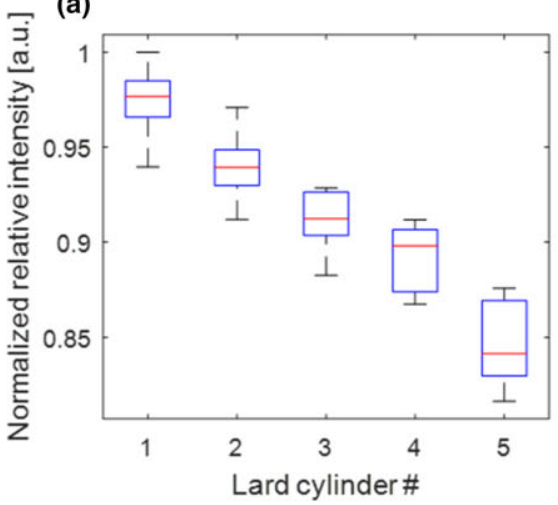

(b)

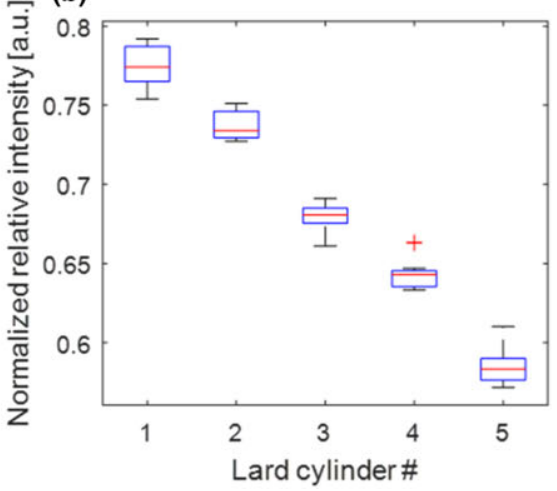

(c)

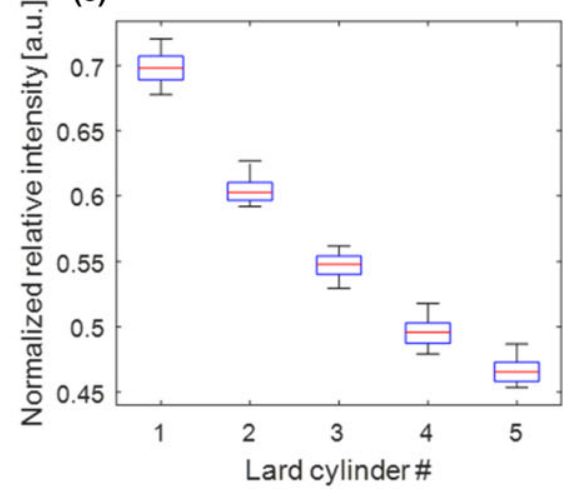

FIG. 8. Normalized relative XA intensities for the five lard cylinders when (a) a $15^{\circ}$ physical wedge, (b) a $30^{\circ}$ physical wedge, or (c) a $45^{\circ}$ physical wedge was used. Ten independent measurements were conducted to compute the mean and the standard deviation for each lard cylinder under each condition. [Color figure can be viewed at wileyonlinelibrary.com] 

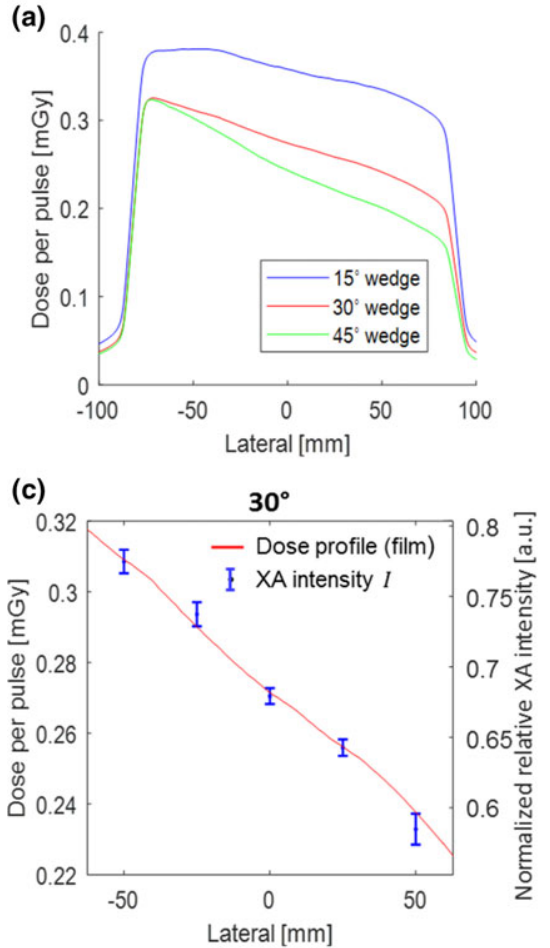
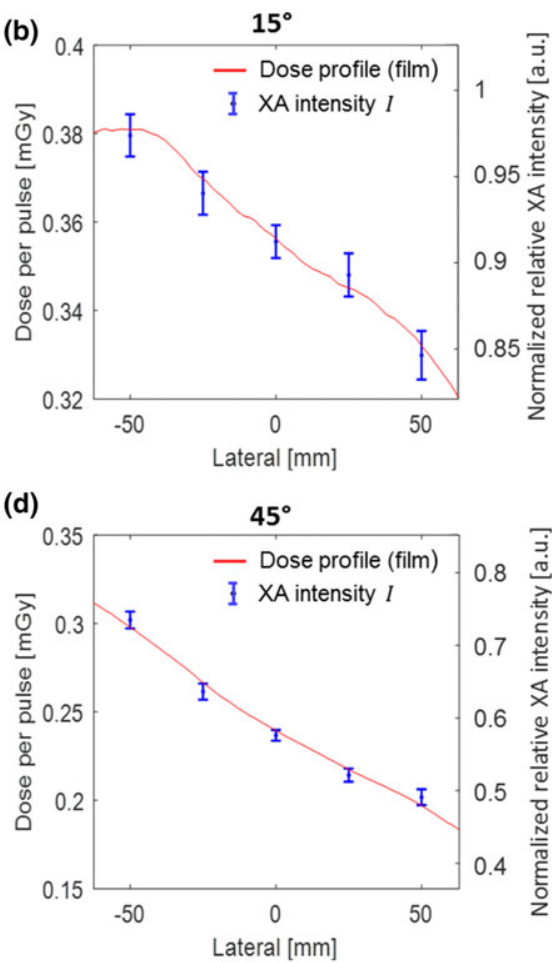

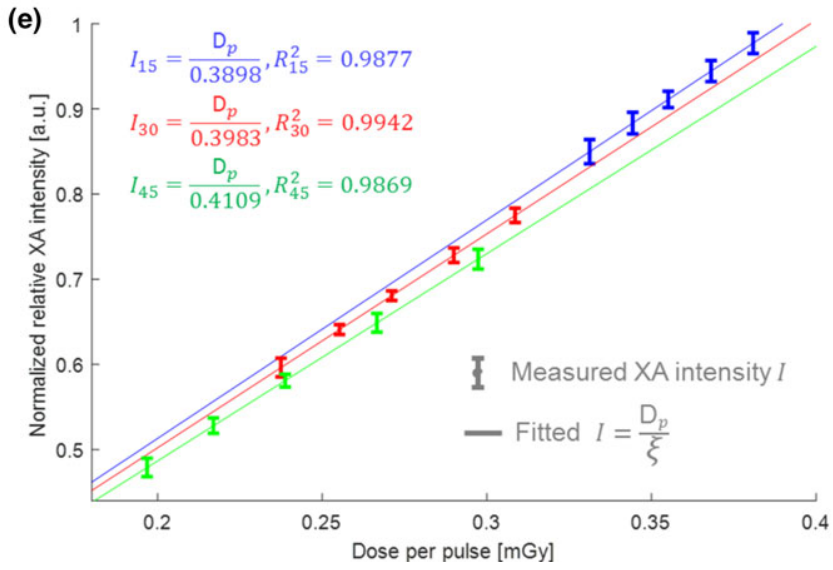

FIG. 9. (a) Dose profiles extracted from the film test results for three physical wedges $\left(15^{\circ}, 30^{\circ}\right.$, and $\left.45^{\circ}\right)$. (b)-(d) Dose per pulse extracted from film profiles shown in (a) matched with the mean value and standard deviation of the normalized relative XA intensities (shown in Fig. 8 ) under the $15^{\circ}, 30^{\circ}$, and $45^{\circ}$ physical wedges, respectively. (e) Correlation between the normalized relative XA intensity and the film measured dose per pulse, including linear fittings follows Eq. (12) for the $15^{\circ}, 30^{\circ}$, and $45^{\circ}$ physical wedges (shown in blue, red, and green, respectively). [Color figure can be viewed at wileyonlinelibrary.com]

Fig. 9(b). Based on the dataset shown in Fig. 8(a), a $t$-test with a null hypothesis that XACT cannot detect the dose difference between the adjacent lard cylinders achieved a significant $P$-value of 0.02 .

Figure 10 displays one representative reconstructed image and the quantified relative XA intensities of the four lard cylinders inside the phantom shown in Fig. 4(a). All measurements were normalized to the mean value of XA intensities of the lard cylinder without being covered by anything except for gel. For the case that the $\mathrm{x}$ ray passed through the air gap before reaching the lard cylinder, the XA intensity from the lard cylinder is about $10 \%$ stronger, as the $\mathrm{x}$ ray experienced lower attenuation inside air compared with gel or water. The XA intensity from the lard cylinder covered by bone is about $20 \%$ weaker, as the $\mathrm{x}$ ray experienced more attenuation while propagating through bone. ${ }^{35}$ The $P$-values calculated from the $t$-tests comparing any two groups were shown in the figure. All $P$-values are smaller than 0.005 except for the $P$-value from the comparison between the muscle group and the gel group due to the fact that muscle and gel have similar $\mathrm{x}$-ray attenuation. The finding from the XACT imaging agrees well with the radiography result shown in Fig. 4(b).

\section{DISCUSSION}

In this work, imaging of several phantoms made from biological tissues was conducted using a prototype XACT 
(a)

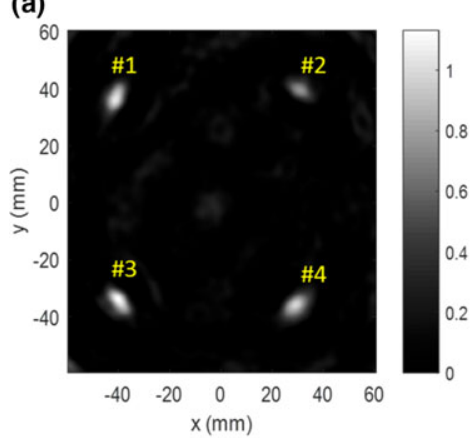

(b)

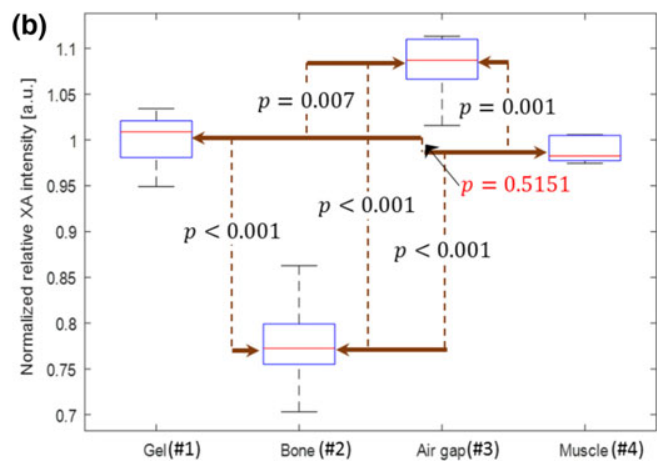

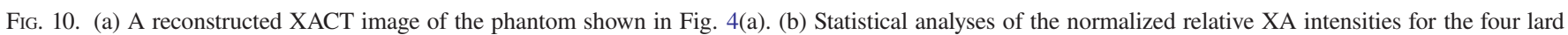
cylinders covered by materials with different X-ray attenuations, including nothing (gel only), bone, air gap, and muscle. The $P$-values from the $t$-tests comparing any of the two groups were evaluated. [Color figure can be viewed at wileyonlinelibrary.com]

system. Because tomographic imaging was achieved via the rotational scan of a single flat transducer around the phantom, only $2 \mathrm{D}$ images in the scanning plane which was perpendicular to the direction of the $\mathrm{x}$-ray beam were reconstructed. In the preparation of the phantoms, therefore, all important features were distributed in a single layer. In the future, however, by scanning around the sample along a $2 \mathrm{D}$ surface, for example, by performing a scan along a spherical surface or a cylindrical surface surrounding the sample, a real 3D image can be reconstructed to evaluate the deposited radiation dose in any voxel.

As demonstrated in Section 3.A, the position and the distribution of the deposited dose inside a phantom can be determined by the acquired XACT image co-registered with the corresponding US image, as the example results shown in Figs. 5 and 6 . The shift of the x-ray beam field against the mimic target inside phantom can be observed through the combined images. It suggests the feasibility of applying $\mathrm{XACT}$ as an add-on to the clinical US to achieve image guidance for radiation therapy. The accuracy of this tracking process is limited by the accuracy of the image registration. In this study, the co-registration was based on the geometric feature of the fat cuboid in XACT image and US image, which took advantage of the extreme contrast between fat and liver in XACT image and was limited by the qualities of acquired XACT image and US image. In in vivo scenario, additional calibration may be necessary so that the acquired XA images and US images can be transformed into the same coordinate system. Since the US and XA signals are both propagate as acoustic waves, their detection sensor can be shared, and the correspondence between their coordinates can be established accurately. In the future, a real-time dual modality system, which can acquire XACT and US images using the same transducer probe driven by the same data acquisition system, may enable improved accuracy for confirming the beam and target alignment for EBRT.

In XACT images, for example, in Fig. 5, strong XA intensities appeared at the beam field boundaries or the interface between the tissues. This is due to the applied reconstruction algorithm and the limited bandwidth of the measuring system which misses some low-frequency components. ${ }^{20}$ To realize the quantitative imaging mentioned in Section 2.B, the acoustic inversion problems including loss of low-frequency components and limited sensor response could be resolved by applying de-convolution methods in the process of image reconstruction. ${ }^{33,36}$ Besides, the $\mathrm{x}$-ray pulse profile should also be taken into consideration, since the current duration of the x-ray beam pulse is too long to be treated as a delta function. ${ }^{37}$ According to the previous studies, ${ }^{21,22}$ limited by the $0.5-\mathrm{MHz}$ central frequency transducer and by the $4-\mu$ s duration of the $\mathrm{x}$-ray pulse, a theoretical spatial resolution of $6 \mathrm{~mm}$ in static image can be achieved and x-ray field shifts of $2 \mathrm{~mm}$ can be determined by our prototype system. When possible, decreasing the duration of the $\mathrm{x}$-ray pulse and maintaining the dose amount per pulse from the Linac could enhance both the spatial resolution and the signal-to-noise ratio (SNR), which is also described in Eq. (10).

As demonstrated in Section 3.B, the intensity inside the $\mathrm{XACT}$ image is related to the energy deposited by the excitation pulse, which implies the possibility of tracking the delivered target dose per pulse independent of the repetition rate of the Linac $(\sim 330 \mathrm{~Hz}$ at the dose rate of $800 \mathrm{MU} / \mathrm{min}$ for $6 \mathrm{MV}$ beam). However, to achieve sufficient SNR, the signals detected for XACT imaging had to be averaged 660 times before being used for image reconstruction. When XA signals need to be collected from 120 scanning positions around the sample, the signal acquisition for each 2D XACT image takes about $10 \mathrm{~min}$ and parts of the radiation pulses were missing due to movement of the transducer between each scanning position. In the future, a circular array probe or a commercially available linear probe driven by a multichannel data acquisition system can be used to replace the circular scan of a single element transducer. In that case, a single XACT image can be formed during a short-time interval, which can be in the order of few seconds or even subseconds. Similar to the works shown in Fig. 9, the intensity of the formed XACT image can be calibrated to the averaged dose deposited by the excitation pulses during that short-time interval following Eq. (12). This would make it possible for tracking the mGy level variation of the dose delivery with high spatial and temporal resolutions, which will be useful for monitoring complex dose delivery techniques such as 
dynamic IMRT and realizing the potentials of (4D) timeresolved intrafraction dose delivery. ${ }^{10}$ The radiation dose at each spatial point will be quantified by the integration of the local XACT intensity over the time intervals. Moreover, as the difference in dose delivery or XA intensity can be determined with a sensitivity of $3 \%$, any changes in the target could be monitored during the treatment.

To evaluate the accuracy of monitoring the dose delivered to the target region, different physical wedges were applied to generate different dose distributions on the target region. The relative dose distributions quantified by XACT were correlated with the dose profiles measured by radiochromic films. All the lard cylinders were modeled to the same geometry and fixed along a line inside the porcine gel which can be treated as a homogeneous background. In this way, a simple back-projection algorithm, which does not consider any attenuations or distortions during acoustic wave propagations, could be applied to extract the initial pressure. The uniformed Grüneisen parameter, thermal efficiency, and density simplified the relation between the delivered dose and the XA intensity described in Eq. (10). However, for clinical implementation, all these parameters need to be considered before truly quantitative evaluation of dose can become possible. In future clinical applications, accessing the tissue properties in the volume of interest could be realized via computed tomography $(\mathrm{CT})$ imaging during the treatment planning. ${ }^{38}$ In this way, quantitative or semiquantitative evaluation and mapping of dose delivery can be achieved.

\section{CONCLUSION}

This work evaluates the feasibility of obtaining relative dose distribution in radiotherapy by performing XACT imaging of soft-tissue phantoms. The study has shown that XACT images of soft-tissue phantoms can be achieved with accuracy in dose measurement of up to $2.9 \%$ at a spatial resolution of $6 \mathrm{~mm}$, which suggests the potential application of XACT imaging for verifying beam alignment with the target tissue and for evaluating accuracy of the dose delivered to the target tissue. Combining XACT imaging with clinical US imaging is a novel in vivo dosimetry approach that has the potential to guide radiotherapy delivery in real time.

\section{ACKNOWLEDGMENTS}

This work was, in part, supported by the University of Michigan Cancer Center fund G017459 and NIH grant CA222215.

\section{CONFLICTS OF INTEREST}

The authors have no conflicts to disclose.

Lei and Zhang contributed equally to this work.

a) Authors to whom correspondence should be addressed. Electronic mails: xdwang@umich.edu; ielnaqa@med.umich.edu.

\section{REFERENCES}

1. Halperin EC, Wazer, DE, Perez, CA, Brady LW. Perez and Brady's principles and practice of radiation oncology, 7th edn. Philadelphia, PA: Wolters Kluwer; 2018.

2. Podgorsak EB. Review of Radiation Oncology Physics: A Handbook for Teachers and Students. Vienna: International Atomic Energy Agency Educational reports series; 2003.

3. Podgoršak EB. Radiation Physics for Medical Physicists. Berlin: Springer; 2006.

4. Hall EJ, Giaccia AJ. Radiobiology for the Radiologist, 7th edn. Philadelphia: Wolters Kluwer Health/Lippincott Williams \& Wilkins; 2012.

5. Lehnert S. Biomolecular Action of Ionizing Radiation. New York: Taylor \& Francis; 2008.

6. Webb S. Intensity-Modulated Radiation Therapy. Boca Raton: CRC Press; 2001.

7. Group IMRTCW. Intensity-modulated radiotherapy: current status and issues of interest. Int J Radiat Oncol Biol Phys. 2001;51:880-914.

8. Sterzing F, Engenhart-Cabillic R, Flentje M, Debus J. Image-guided radiotherapy. Deutsch Aerztebl Int. 2011;108:274-280.

9. Timmerman RD, Xing L. Image-Guided and Adaptive Radiation Therapy. Philadelphia, PA: Lippincott Williams \& Wilkins; 2012.

10. Mijnheer B, Olaciregui-Ruiz I, Rozendaal R, et al. 3D EPID-based in vivo dosimetry for IMRT and VMAT. Paper presented at: $J$ Phys: Confe Ser. 2013;444:012011.

11. Mijnheer B, Beddar S, Izewska J, Reft C. In vivo dosimetry in external beam radiotherapy. Med Phys. 2013;40:070903.

12. Huyskens DP. Practical Guidelines for the Implementation of In Vivo Dosimetry with Diodes in External Radiotherapy with Photon Beams (Entrance Dose). Barcelona: ESTRO; 2001.

13. Yorke E, Alecu R, Ding L, et al. Diode in vivo dosimetry for patients receiving external beam radiation therapy. Report of Task Group; 2005, 62.

14. Ramaseshan R, Kohli K, Zhang T, et al. Performance characteristics of a microMOSFET as an in vivo dosimeter in radiation therapy. Phys Med Biol. 2004;49:4031.

15. Chuang CF, Verhey LJ, Xia P. Investigation of the use of MOSFET for clinical IMRT dosimetric verification. Med Phys. 2002;29:1109-1115.

16. van Elmpt W, McDermott L, Nijsten S, Wendling M, Lambin P, Mijnheer B. A literature review of electronic portal imaging for radiotherapy dosimetry. Radiother Oncol. 2008;88:289-309.

17. Mans A, Remeijer P, Olaciregui-Ruiz I, et al. 3D Dosimetric verification of volumetric-modulated arc therapy by portal dosimetry. Radiother Oncol. 2010;94:181-187.

18. Jornet N, Carrasco P, Jurado D, Ruiz A, Eudaldo T, Ribas M. Comparison study of MOSFET detectors and diodes for entrance in vivo dosimetry in 18 MV x-ray beams. Med Phys. 2004;31:2534-2542.

19. Wang LV, Hu S. Photoacoustic tomography: in vivo imaging from organelles to organs. Science. 2012;335:1458-1462.

20. Xiang L, Han B, Carpenter C, Pratx G, Kuang Y, Xing L. X-ray acoustic computed tomography with pulsed $\mathrm{X}$-ray beam from a medical linear accelerator. Med Phys. 2013;40:010701.

21. Hickling S, Lei H, Hobson M, Léger P, Wang X, El Naqa I. Experimental evaluation of $\mathrm{X}$-ray acoustic computed tomography for radiotherapy dosimetry applications. Med Phys. 2017;44:608-617.

22. Hickling S, Hobson M, Naqa IE. Characterization of X-ray acoustic computed tomography for applications in radiotherapy dosimetry. IEEE Trans Radiat Plasma Med Sci. 2018;2:337-344.

23. Tang S, Nguyen DH, Zarafshani A, et al. X-ray-induced acoustic computed tomography with an ultrasound transducer ring-array. Appl Phys Lett. 2017;110:103504.

24. Shanshan T, Kai Y, Yong C, Liangzhong X. X-ray-induced acoustic computed tomography for 3D breast imaging: a simulation study. Med Phys. 2018;45:1662-1672.

25. Gunderson LL. Clinical Radiation Oncology. Philadelphia: Elsevier Health Sciences; 2015.

26. Fang Y, Vasil'ev AN, Mikhailin VV. Theory of X-ray photoacoustic spectroscopy. Appl Phys A. 1995;60:333-341.

27. Kruger RA, Liu P, Fang YR, Appledorn CR. Photoacoustic ultrasound (PAUS) - reconstruction tomography. Med Phys. 1995;22:1605-1609.

28. Welch AJ, Van Gemert MJ. Optical-Thermal Response of Laser-Irradiated Tissue. Vol. 2. Heidelberg: Springer; 2011. 
29. Kainz K. Radiation oncology physics: a handbook for teachers and students. Med Phys. 2006;33:1920-1920.

30. Hickling S, Léger P, El Naqa I. On the detectability of acoustic waves induced following irradiation by a radiotherapy linear accelerator. IEEE Trans Ultrason Ferroelectr Freq Control. 2016;63:683-690.

31. Zhou Y, Yao J, Wang LV. Tutorial on photoacoustic tomography. $J$ Biomed Opt. 2016;21:061007.

32. Xia J, Yao J, Wang LV. Photoacoustic tomography: principles and advances. Electromagn Waves (Cambridge, Mass). 2014;147:1-22.

33. Cox BT, Laufer JG, Beard PC. The challenges for quantitative photoacoustic imaging. Paper presented at: SPIE BiOS; 2009.

34. Xu M, Wang LV. Universal back-projection algorithm for photoacoustic computed tomography. Phys Rev E. 2005;71:016706.
35. Hubbell JH, Seltzer SM. Tables of X-ray mass attenuation coefficients and mass energy-absorption coefficients $1 \mathrm{keV}$ to $20 \mathrm{MeV}$ for elements $Z=1$ to 92 and 48 additional substances of dosimetric interest. National Inst. of Standards and Technology-PL, Gaithersburg, MD (United States). Ionizing Radiation Div.;1995.

36. Pramanik M. Improving tangential resolution with a modified delayand-sum reconstruction algorithm in photoacoustic and thermoacoustic tomography. JOSA A. 2014;31:621-627.

37. Yi W, Da X, Yaguang Z, Qun C. Photoacoustic imaging with deconvolution algorithm. Phys Med Biol. 2004;49:3117.

38. Johns PC, Yaffe MJ. X-ray characterisation of normal and neoplastic breast tissues. Phys Med Biol. 1987;32:675. 